Supporting Information

\title{
Discovery of Arylsulfonamides as Dual Orexin
}

\section{Receptor Agonists}

Dehui Zhang, ${ }^{a}$ David A. Perrey, ${ }^{a}$ Ann M. Decker,${ }^{a}$ Tiffany L. Langston, ${ }^{a}$ Vijayakumar Mavanji, ${ }^{b}$

Danni L. Harris, ${ }^{a}$ Catherine M. Kotz, ${ }^{b, c, d}$ and Yanan Zhang ${ }^{a, *}$

a Research Triangle Institute, Research Triangle Park, North Carolina 27709

${ }^{\mathrm{b}}$ Research Service, Veterans Affairs Health Care System, Minneapolis, MN 55417

${ }^{c}$ Department of Integrative Biology and Physiology, University of Minnesota, Minneapolis, MN 55455

${ }^{\mathrm{d}}$ Geriatric, Research, Education and Clinical Center, Minneapolis Veterans Affairs Health Care System, Minneapolis, MN 55417

* Corresponding Author: yzhang@rti.org

\section{Table of Contents}

1. HPLC purity and retention time S2

2. HPLC traces of representative target compounds $\quad$ S4

3. ${ }^{11} \mathrm{H}$ and ${ }^{13} \mathrm{C}$ NMR of $\mathbf{4 0} \quad \mathrm{S} 19$ 
Compound purity by HPLC

\begin{tabular}{|c|c|c|}
\hline Compound & Retention time (min) & Purity (\%) \\
\hline 2 & 12.421 & 98.3 \\
\hline 6 & 13.372 & 98.4 \\
\hline 7 & 13.576 & $>99$ \\
\hline 8 & 12.923 & $>99$ \\
\hline 9 & 13.105 & 99.0 \\
\hline 10 & 11.049 & 96.8 \\
\hline 11 & 11.084 & 95.0 \\
\hline 12 & 15.528 & 99.0 \\
\hline 13 & 11.274 & 95.1 \\
\hline 14 & 12.154 & 96.9 \\
\hline 15 & 15.874 & $>99$ \\
\hline 16 & 14.823 & 95.1 \\
\hline 17 & 13.976 & 98.1 \\
\hline 18 & 11.910 & 95.2 \\
\hline 19 & 13.367 & 95.0 \\
\hline 20 & 12.836 & 95.6 \\
\hline 21 & 14.411 & 96.2 \\
\hline 22 & 12.928 & $>99$ \\
\hline 23 & 14.793 & $>99$ \\
\hline 24 & 12.713 & 98.3 \\
\hline 25 & 12.302 & 98.9 \\
\hline 26 & 12.720 & $>99$ \\
\hline 27 & 13.467 & $>99$ \\
\hline 28 & 13.683 & 98.7 \\
\hline 29 & 13.635 & 98.7 \\
\hline 30 & 14.107 & 98.9 \\
\hline 31 & 13.028 & 95.3 \\
\hline 32 & 16.023 & 96.2 \\
\hline 33 & 12.592 & 95.6 \\
\hline 34 & 12.506 & 98.4 \\
\hline 35 & 13.091 & 96.1 \\
\hline
\end{tabular}




\begin{tabular}{lll}
\hline $\mathbf{3 6}$ & 15.585 & $>99$ \\
\hline $\mathbf{3 7}$ & 12.767 & 98.6 \\
\hline $\mathbf{3 8}$ & 12.642 & $>99$ \\
\hline $\mathbf{3 9}$ & 12.517 & 98.2 \\
\hline $\mathbf{4 0}$ & 12.497 & $>99$ \\
\hline $\mathbf{4 1}$ & 12.653 & 98.1 \\
\hline $\mathbf{4 2}$ & 12.395 & 97.8 \\
\hline $\mathbf{4 3}$ & 13.951 & 98.5 \\
\hline $\mathbf{4 4}$ & 12.455 & $>99$ \\
\hline
\end{tabular}


WalkUpReport

\begin{tabular}{|llll|}
\hline & \multicolumn{1}{c|}{ S A M P E } & I N F O R M A T I O N \\
\hline \hline Sample Name: & $13937-054$ & Acquired By: & walkup \\
Sample Type: & Unknown & Sample Set Name: & DehuiDavidHPLC \\
Vial: & 1 & Acq. Method Set: & MethodSetAB5T95B15Min \\
Injection \#: & 1 & Processing Method: & 220W280W15MIN \\
Injection Volume: & 20.00 ul & Channel Name: & W2996 280.0nm-4.0 \\
Run Time: & 30.0 Minutes & Proc. Chnl. Descr.: & W2996 PDA 280.0 nm at 4.0 \\
& & & \\
Date Acquired: & $1 / 27 / 20217: 35: 50$ PM EST & & \\
Date Processed: & $4 / 2 / 20213: 27: 05$ PM EST & & \\
\hline
\end{tabular}

\section{Auto-Scaled Chromatogram}

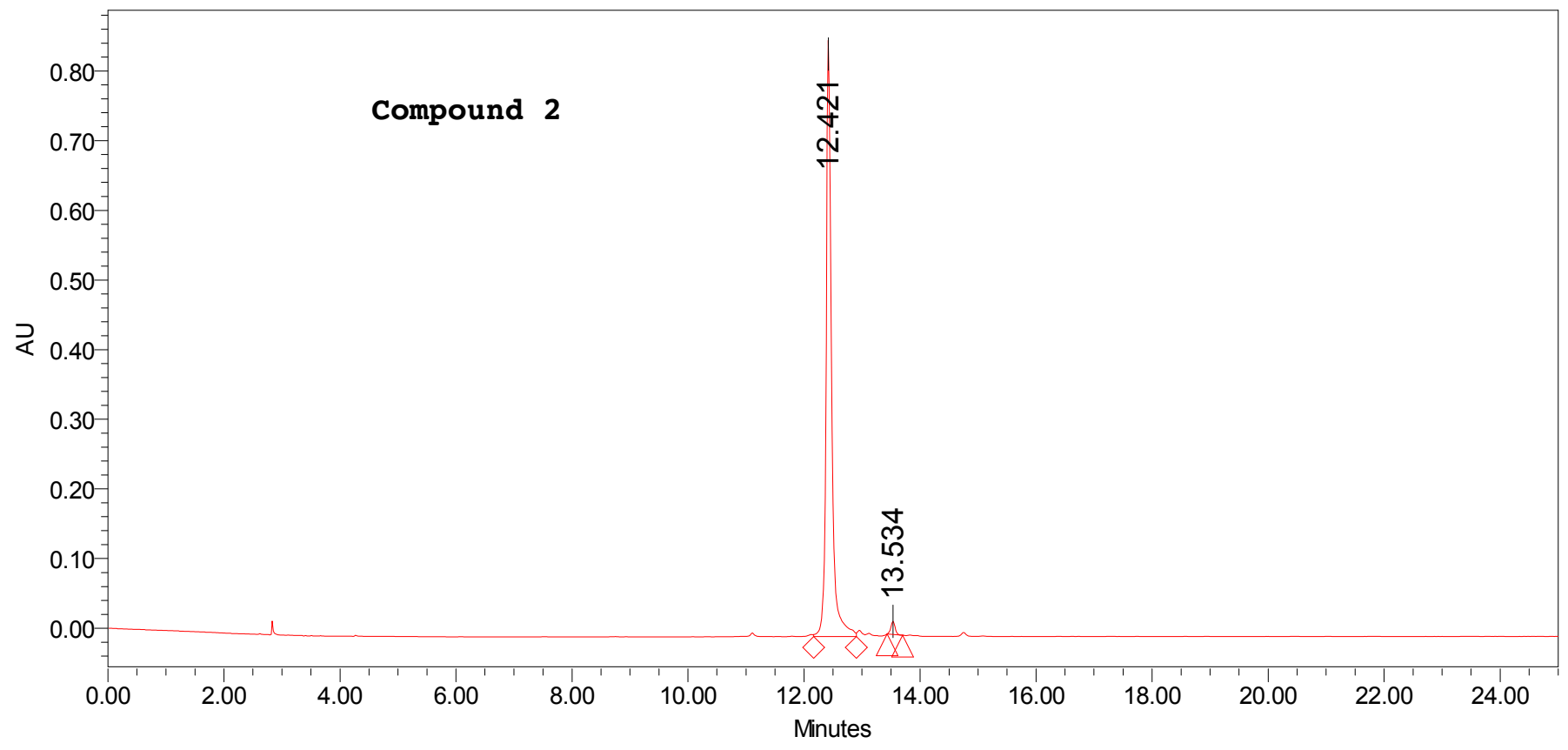

\section{Peak Results}

\begin{tabular}{|r|c|r|r|r|}
\hline & RT & Area & \% Area & Height \\
\hline 1 & 12.421 & 5498165 & 98.29 & 851114 \\
\hline 2 & 13.534 & 95466 & 1.71 & 18474 \\
\hline
\end{tabular}




\begin{tabular}{|llll|}
\hline & \multicolumn{1}{c|}{ S A M P L E } & I N F O R M A T I O N \\
\hline \hline Sample Name: & 13937-081 & Acquired By: & walkup \\
Sample Type: & Unknown & Sample Set Name: & DehuiDavidHPLC \\
Vial: & 5 & Acq. Method Set: & MethodSetAB5T95B15Min \\
Injection \#: & 1 & Processing Method: & 220W280W15MIN \\
Injection Volume: & 20.00 ul & Channel Name: & W2996 280.0nm-4.0 \\
Run Time: & 30.0 Minutes & Proc. Chnl. Descr.: & W2996 PDA 280.0 nm at 4.0 \\
& & & \\
Date Acquired: & 1/27/2021 9:39:58 PM EST & & \\
Date Processed: & $4 / 2 / 20213: 59: 12$ PM EST & & \\
\hline
\end{tabular}

Auto-Scaled Chromatogram

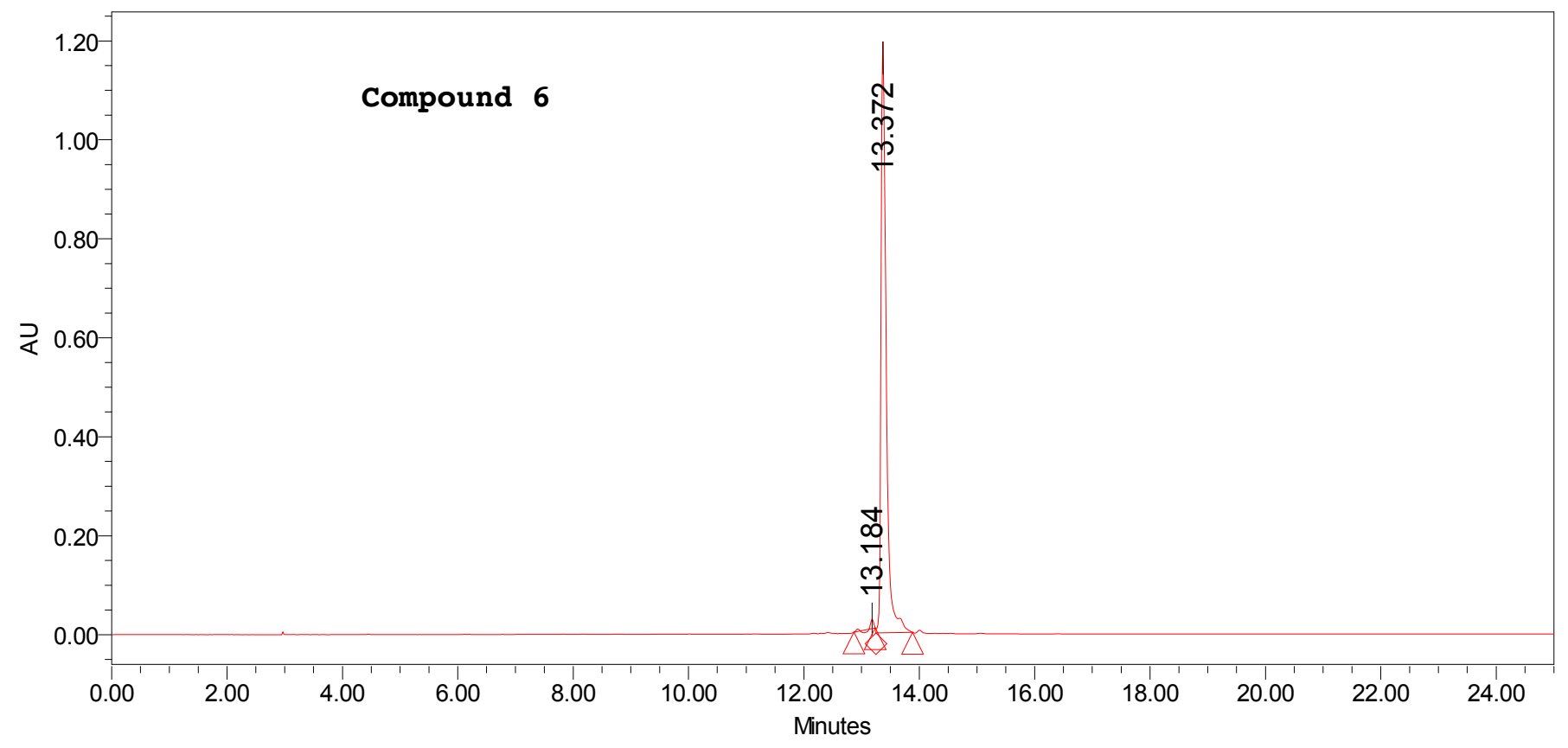

Peak Results

\begin{tabular}{|r|c|r|r|r|}
\hline & RT & Area & \% Area & Height \\
\hline 1 & 13.184 & 118486 & 1.58 & 18186 \\
\hline 2 & 13.372 & 7396048 & 98.42 & 1204050 \\
\hline
\end{tabular}


WalkUpReport

\begin{tabular}{|llll|}
\hline & \multicolumn{1}{c|}{ S A M P E } & I N F O R M A T I O N \\
\hline \hline Sample Name: & 13937-074 & Acquired By: & walkup \\
Sample Type: & Unknown & Sample Set Name: & DehuiDavidHPLC \\
Vial: & 2 & Acq. Method Set: & MethodSetAB5T95B15Min \\
Injection \#: & 1 & Processing Method: & 220W280W15MIN \\
Injection Volume: & 20.00 ul & Channel Name: & W2996 280.0nm-4.0 \\
Run Time: & 30.0 Minutes & Proc. Chnl. Descr.: & W2996 PDA 280.0 nm at 4.0 \\
& & & \\
Date Acquired: & $1 / 27 / 20218: 06: 52$ PM EST & & \\
Date Processed: & $4 / 2 / 20214: 00: 39$ PM EST & & \\
\hline
\end{tabular}

Auto-Scaled Chromatogram

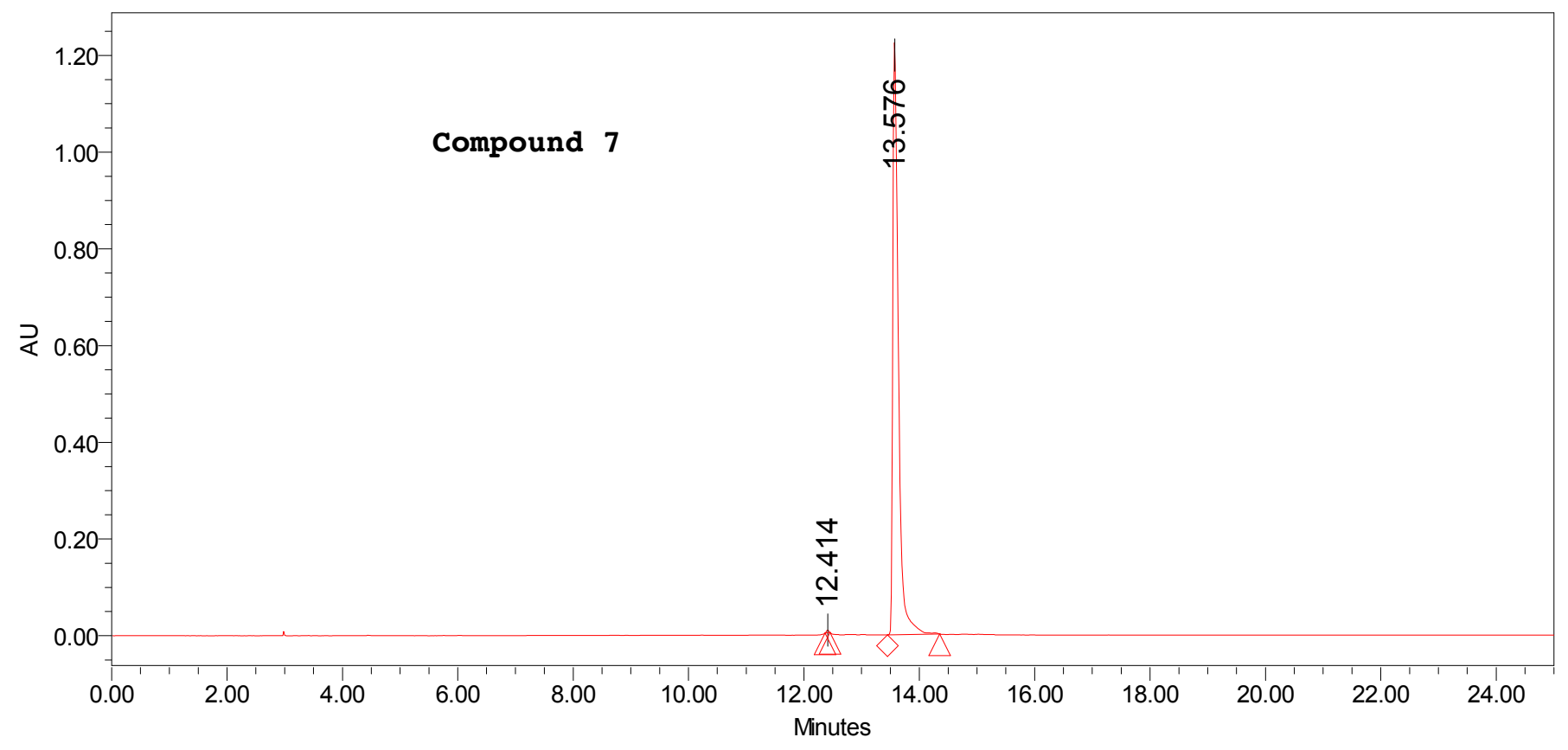

Peak Results

\begin{tabular}{|r|c|r|r|r|}
\hline & RT & \multicolumn{1}{|c|}{ Area } & \% Area & Height \\
\hline 1 & 12.414 & 15531 & 0.19 & 5298 \\
\hline 2 & 13.576 & 8317200 & 99.81 & 1256770 \\
\hline
\end{tabular}




\begin{tabular}{|llll|}
\hline & \multicolumn{1}{c|}{ S A M P L E } & I N F O R M A T I O N \\
\hline \hline Sample Name: & $14045-085$ & Acquired By: & walkup \\
Sample Type: & Unknown & Sample Set Name: & DehuiDavidHPLC \\
Vial: & 12 & Acq. Method Set: & MethodSetAB5T95B15Min \\
Injection \#: & 1 & Processing Method: & 220W280W15MIN \\
Injection Volume: & 20.00 ul & Channel Name: & W2996 280.0nm-4.0 \\
Run Time: & 30.0 Minutes & Proc. Chnl. Descr.: & W2996 PDA 280.0 nm at 4.0 \\
& & & \\
Date Acquired: & 1/28/2021 1:17:08 AM EST & & \\
Date Processed: & $4 / 2 / 20214: 03: 30$ PM EST & & \\
\hline
\end{tabular}

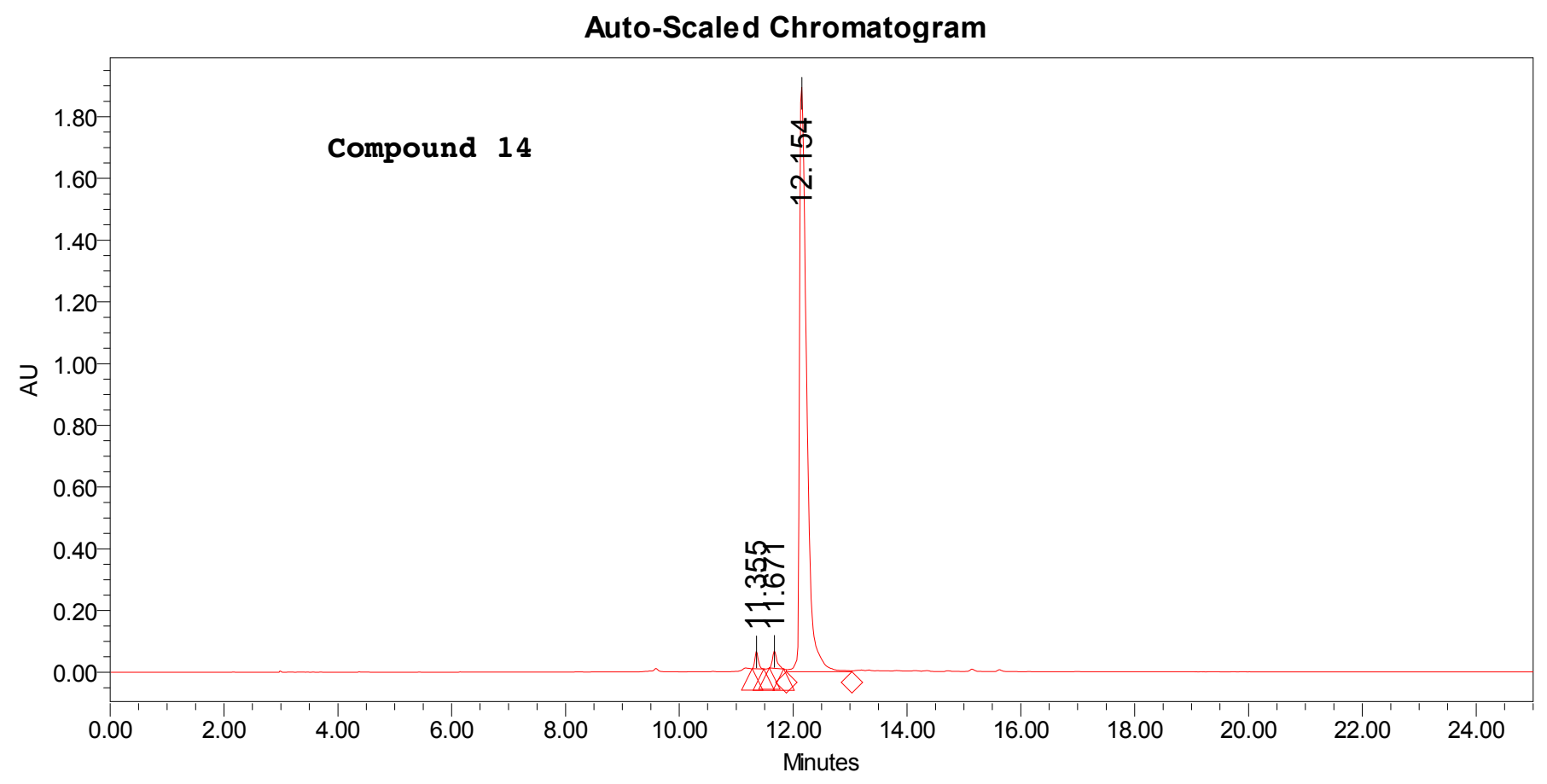

Peak Results
\begin{tabular}{|c|c|r|r|r|}
\hline & RT & Area & $\%$ Area & Height \\
\hline 1 & 11.355 & 233116 & 1.35 & 54519 \\
\hline 2 & 11.671 & 300555 & 1.74 & 54890 \\
\hline 3 & 12.154 & 16725811 & 96.91 & 1914526 \\
\hline
\end{tabular}




\begin{tabular}{|llll|}
\hline & \multicolumn{1}{c|}{ S A M P L E } & I N F O R M A T I O N \\
\hline \hline Sample Name: & $14045-093$ & Acquired By: & walkup \\
Sample Type: & Unknown & Sample Set Name: & DehuiDavidHPLC \\
Vial: & 13 & Acq. Method Set: & MethodSetAB5T95B15Min \\
Injection \#: & 1 & Processing Method: & 220W280W15MIN \\
Injection Volume: & 20.00 ul & Channel Name: & W2996 280.0nm-4.0 \\
Run Time: & 30.0 Minutes & Proc. Chnl. Descr.: & W2996 PDA 280.0 nm at 4.0 \\
& & & \\
Date Acquired: & 1/28/2021 1:48:11 AM EST & & \\
Date Processed: & $4 / 2 / 20214: 07: 03$ PM EST & & \\
\hline
\end{tabular}

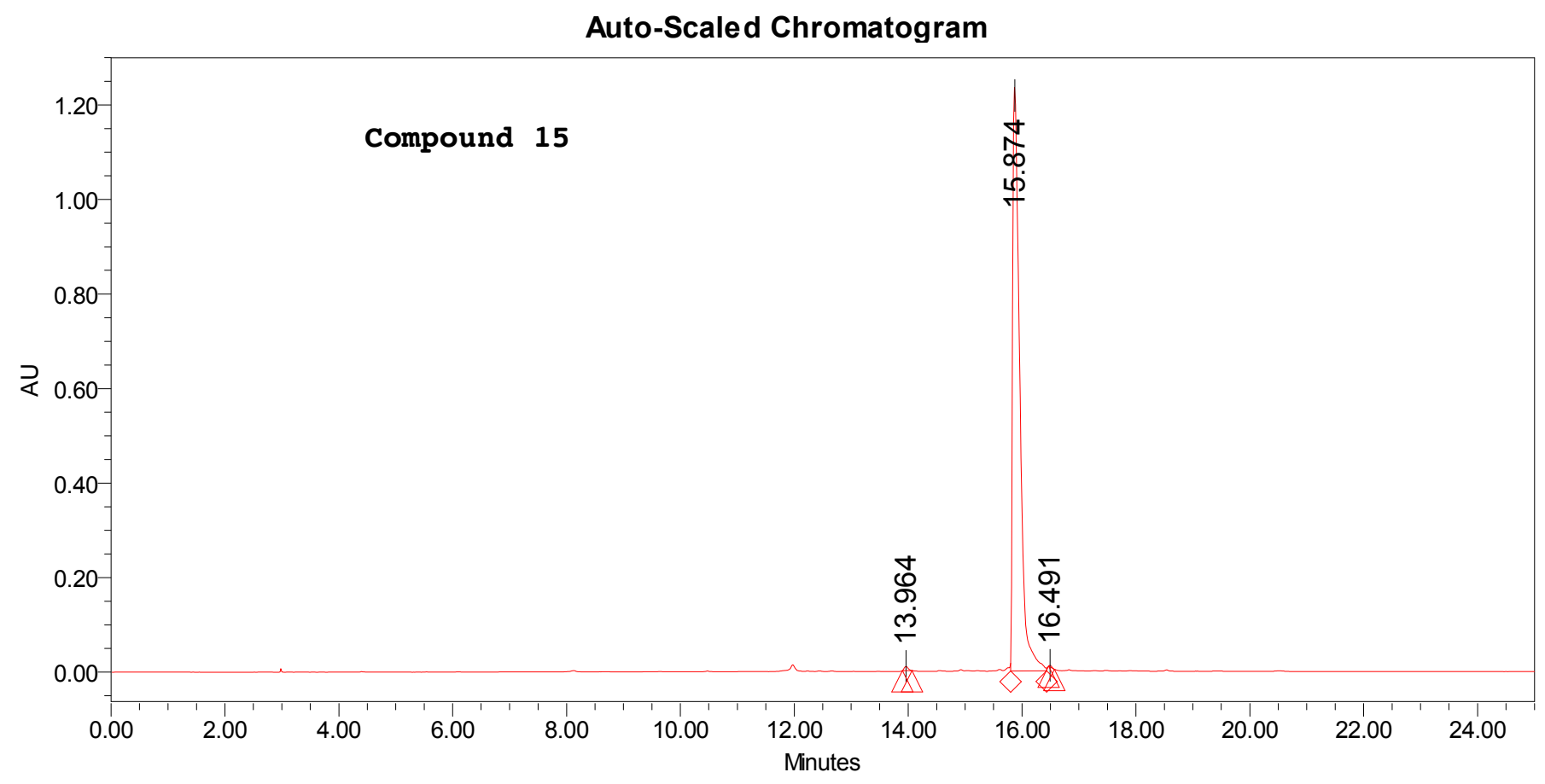

Peak Results
\begin{tabular}{|l|c|r|r|r|}
\hline & RT & \multicolumn{1}{c|}{ Area } & $\%$ Area & \multicolumn{1}{c|}{ Height } \\
\hline 1 & 13.964 & 42013 & 0.39 & 8978 \\
\hline 2 & 15.874 & 10856650 & 99.51 & 1266499 \\
\hline 3 & 16.491 & 11883 & 0.11 & 3511 \\
\hline
\end{tabular}




\begin{tabular}{|llll|}
\hline & \multicolumn{1}{c|}{ S A M P L E } & I N F O R M A T I O N \\
\hline \hline Sample Name: & DZ14227-23 & Acquired By: & walkup \\
Sample Type: & Unknown & Sample Set Name: & DZ142272325 \\
Vial: & 25 & Acq. Method Set: & MethodSetAB5T95B15Min \\
Injection \#: & 1 & Processing Method: & 220W280W15MIN \\
Injection Volume: & 20.00 ul & Channel Name: & W2996 280.0nm-4.0 \\
Run Time: & 30.0 Minutes & Proc. Chnl. Descr.: & W2996 PDA 280.0 nm at 4.0 \\
& & & \\
Date Acquired: & $9 / 1 / 20207: 35: 26$ PM EDT & & \\
Date Processed: & $4 / 2 / 20214: 27: 47$ PM EST & & \\
\hline
\end{tabular}

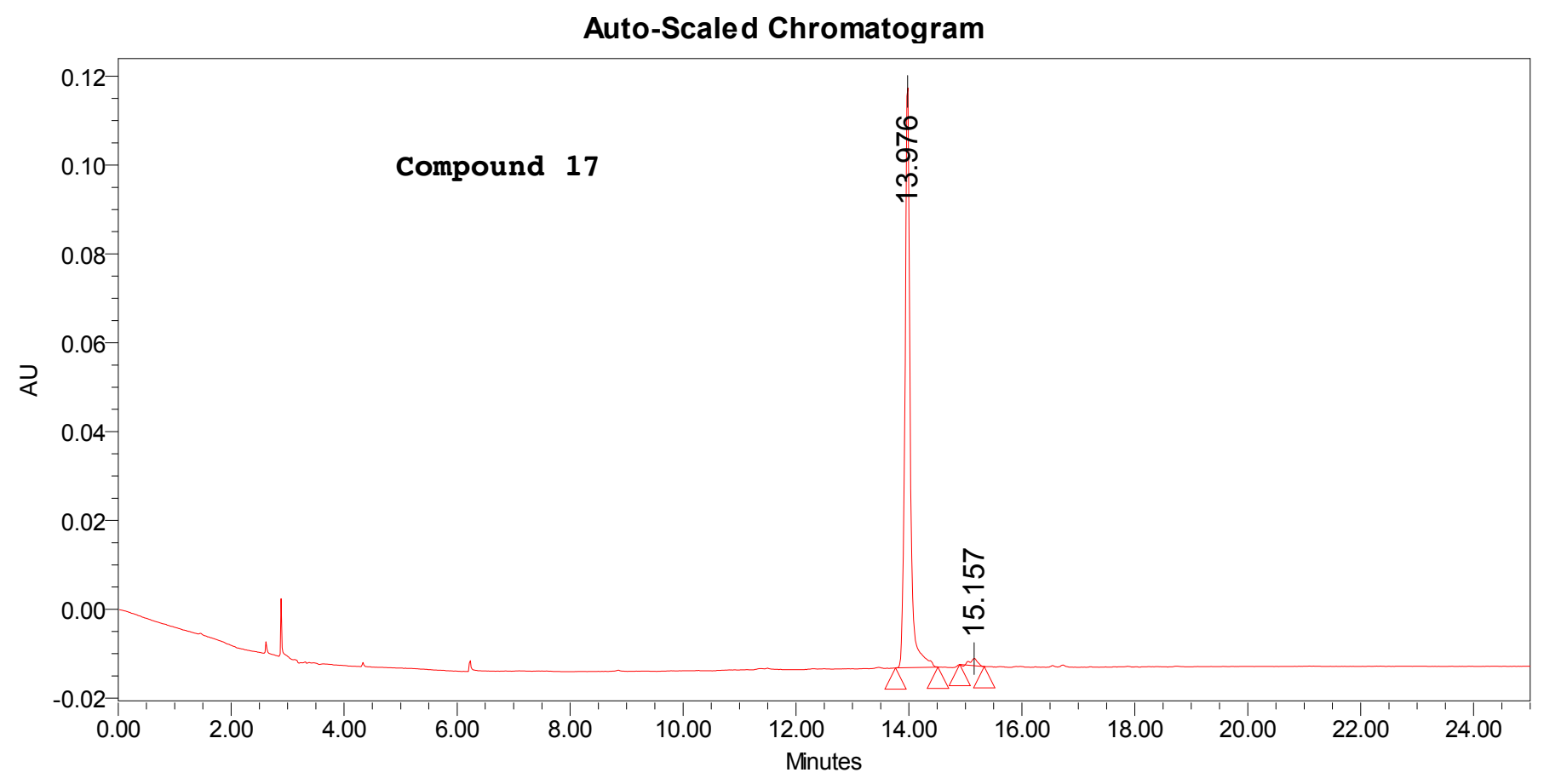

Peak Results
\begin{tabular}{|l|c|r|r|r|}
\hline & RT & Area & $\%$ Area & Height \\
\hline 1 & 13.976 & 841421 & 98.10 & 130310 \\
\hline 2 & 15.157 & 16278 & 1.90 & 1659 \\
\hline
\end{tabular}




\begin{tabular}{|llll|}
\hline & \multicolumn{1}{c|}{ S A M P L E } & I N F O R M A T I O N \\
\hline \hline Sample Name: & $14045-181$ & Acquired By: & walkup \\
Sample Type: & Unknown & Sample Set Name: & DehuiDavidHPLC \\
Vial: & 17 & Acq. Method Set: & MethodSetAB5T95B15Min \\
Injection \#: & 1 & Processing Method: & 220W280W15MIN \\
Injection Volume: & 20.00 ul & Channel Name: & W2996 280.0nm-4.0 \\
Run Time: & 30.0 Minutes & Proc. Chnl. Descr.: & W2996 PDA 280.0 nm at 4.0 \\
& & & \\
Date Acquired: & 1/28/2021 3:52:18 AM EST & & \\
Date Processed: & $4 / 2 / 20214: 13: 14$ PM EST & & \\
\hline
\end{tabular}

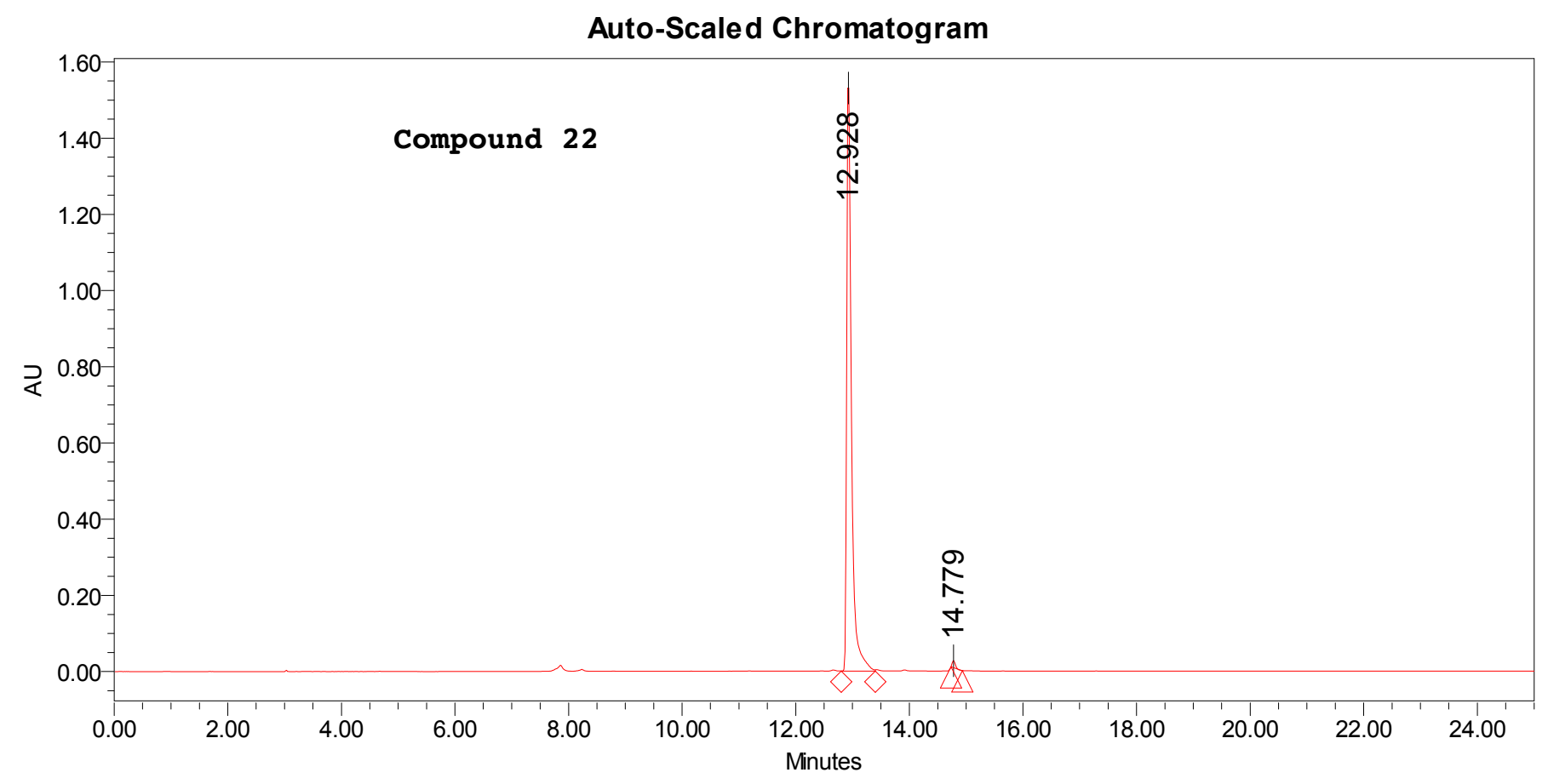

Peak Results
\begin{tabular}{|l|c|r|r|r|}
\hline & RT & \multicolumn{1}{c|}{ Area } & $\%$ Area & \multicolumn{1}{c|}{ Height } \\
\hline 1 & 12.928 & 9081791 & 99.21 & 1563802 \\
\hline 2 & 14.779 & 72481 & 0.79 & 18104 \\
\hline
\end{tabular}


WalkUpReport

\begin{tabular}{|c|c|c|c|}
\hline \multicolumn{2}{|r|}{ SA M P L E } & \multicolumn{2}{|c|}{ I NFORMATION } \\
\hline Sample Name: & $14171-57$ & Acquired By: & walkup \\
\hline Sample Type: & Unknown & Sample Set Name: & DZOXAgonist paper \\
\hline Vial: & 98 & Acq. Method Set: & MethodSetAB5T95B15Min \\
\hline Injection \#: & 1 & Processing Method: & 220W280W15MIN \\
\hline Injection Volume: & $10.00 \mathrm{ul}$ & Channel Name: & W2996 280.0nm-4.0 \\
\hline Run Time: & 30.0 Minutes & Proc. Chnl. Descr.: & W2996 PDA $280.0 \mathrm{~nm}$ at 4.0 \\
\hline Date Acquired: & 9/4/2020 7:42:29 PM EDT & & \\
\hline Date Processed: & 4/2/2021 4:29:40 PM EST & & \\
\hline
\end{tabular}

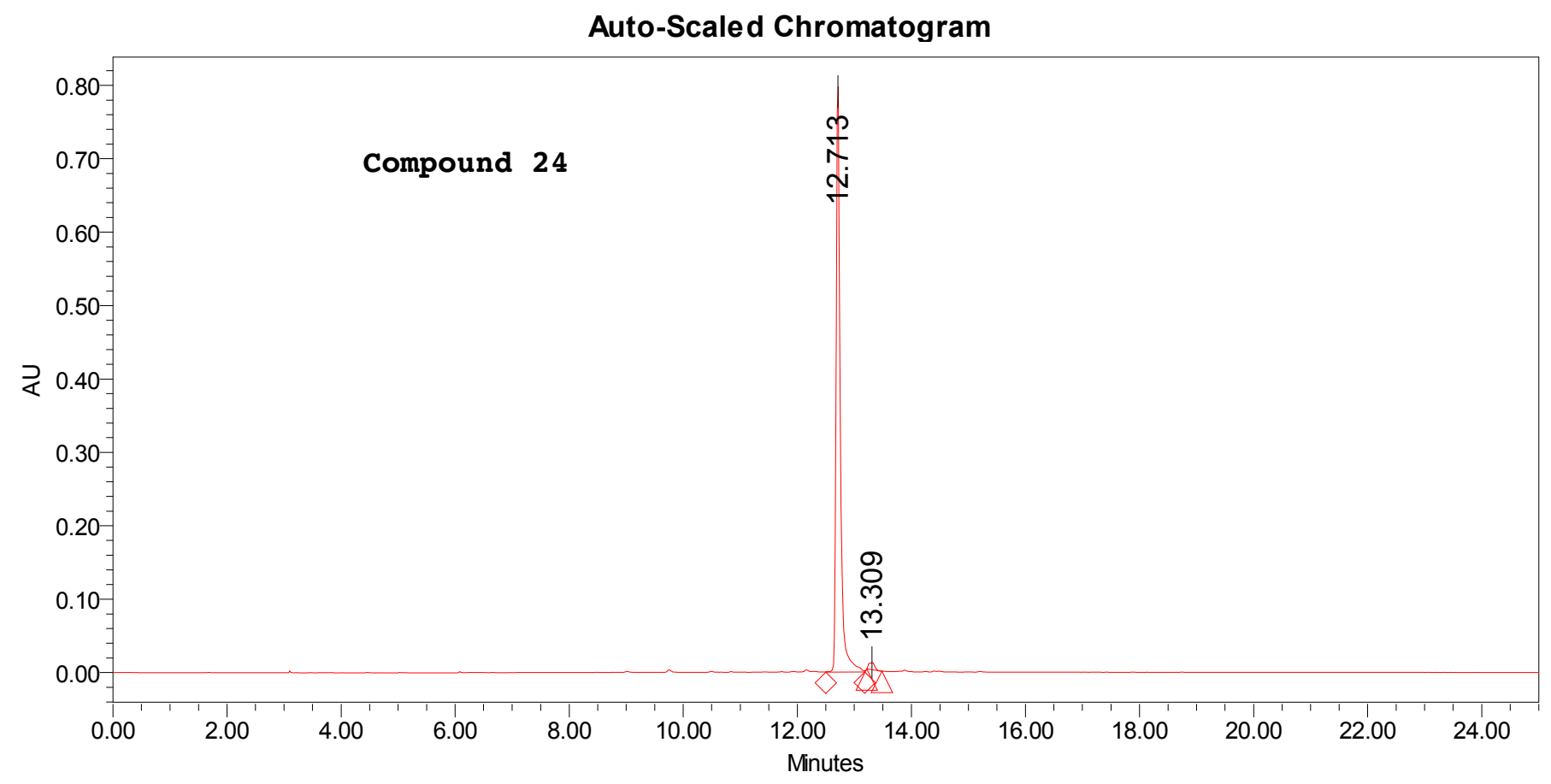

Peak Re sults
\begin{tabular}{|l|c|r|r|r|}
\hline & RT & \multicolumn{1}{c|}{ Area } & $\%$ Area & Height \\
\hline 1 & 12.713 & 4034950 & 98.29 & 785179 \\
\hline 2 & 13.309 & 70221 & 1.71 & 9906 \\
\hline
\end{tabular}

Reported by User: walkup Report Method: WalkUpReport Report Method ID 1018 Page: 1 of 1
Project Name: MedChemlMedChem2020-09

Date Printed:

$4 / 2 / 2021$ 
WalkUpReport

\begin{tabular}{|llll|}
\hline & \multicolumn{1}{c|}{ S A M P L E } & I N F O R M A T I O N \\
\hline \hline Sample Name: & $14171-62$ & Acquired By: & walkup \\
Sample Type: & Unknown & Sample Set Name: & DZ1417162OXpaper \\
Vial: & 1 & Acq. Method Set: & MethodSetAB5T95B15Min \\
Injection \#: & 1 & Processing Method: & 220W280W15MIN \\
Injection Volume: & 10.00 ul & Channel Name: & W2996 280.0nm-4.0 \\
Run Time: & 30.0 Minutes & Proc. Chnl. Descr.: & W2996 PDA 280.0 nm at 4.0 \\
& & & \\
Date Acquired: & $9 / 5 / 20203: 47: 42$ PM EDT & & \\
Date Processed: & $4 / 2 / 20214: 31: 33$ PM EST & & \\
\hline
\end{tabular}

Auto-Scaled Chromatogram

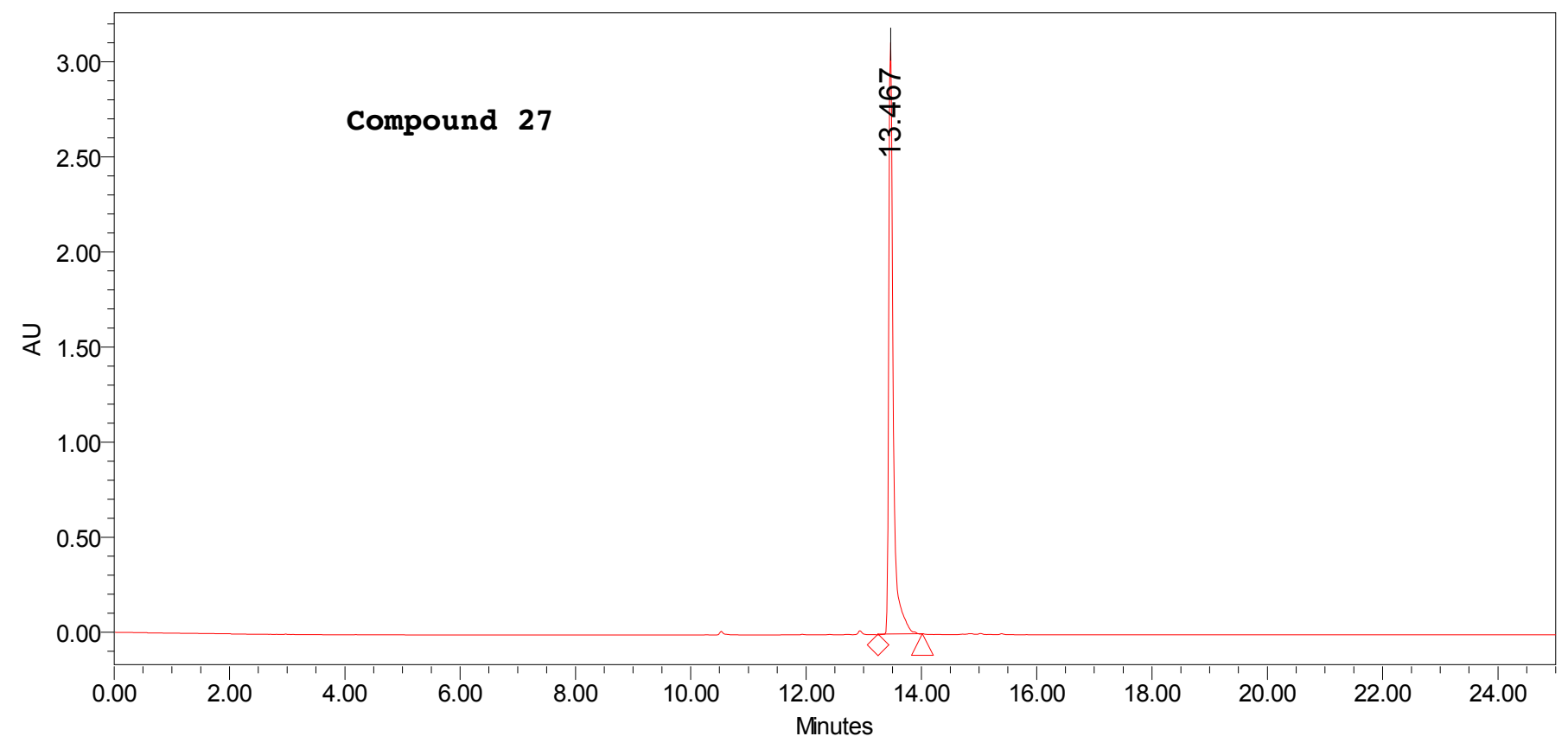

\section{Peak Results}

\begin{tabular}{|c|c|c|c|c|}
\hline & RT & Area & $\%$ Area & Height \\
\hline 1 & 13.467 & 16853280 & 100.00 & 3135157 \\
\hline
\end{tabular}

Reported by User: walkup Report Method: WalkUpReport Report Method ID 1018 Page: 1 of 1
Project Name: MedChemlMedChem2020-09

Date Printed:

$4 / 2 / 2021$ 
WalkUpReport

\begin{tabular}{|llll|}
\hline & \multicolumn{1}{c|}{ S A M P E } & I N F O R M A T I O N \\
\hline \hline Sample Name: & $14171-65$ & Acquired By: & walkup \\
Sample Type: & Unknown & Sample Set Name: & DZOXAgonist paper \\
Vial: & 100 & Acq. Method Set: & MethodSetAB5T95B15Min \\
Injection \#: & 1 & Processing Method: & 220W280W15MIN \\
Injection Volume: & 10.00 ul & Channel Name: & W2996 280.0nm-4.0 \\
Run Time: & 30.0 Minutes & Proc. Chnl. Descr.: & W2996 PDA 280.0 nm at 4.0 \\
& & & \\
Date Acquired: & $9 / 4 / 2020$ 8:44:24 PM EDT & & \\
Date Processed: & $4 / 2 / 20214: 32: 39$ PM EST & & \\
\hline
\end{tabular}

Auto-Scaled Chromatogram

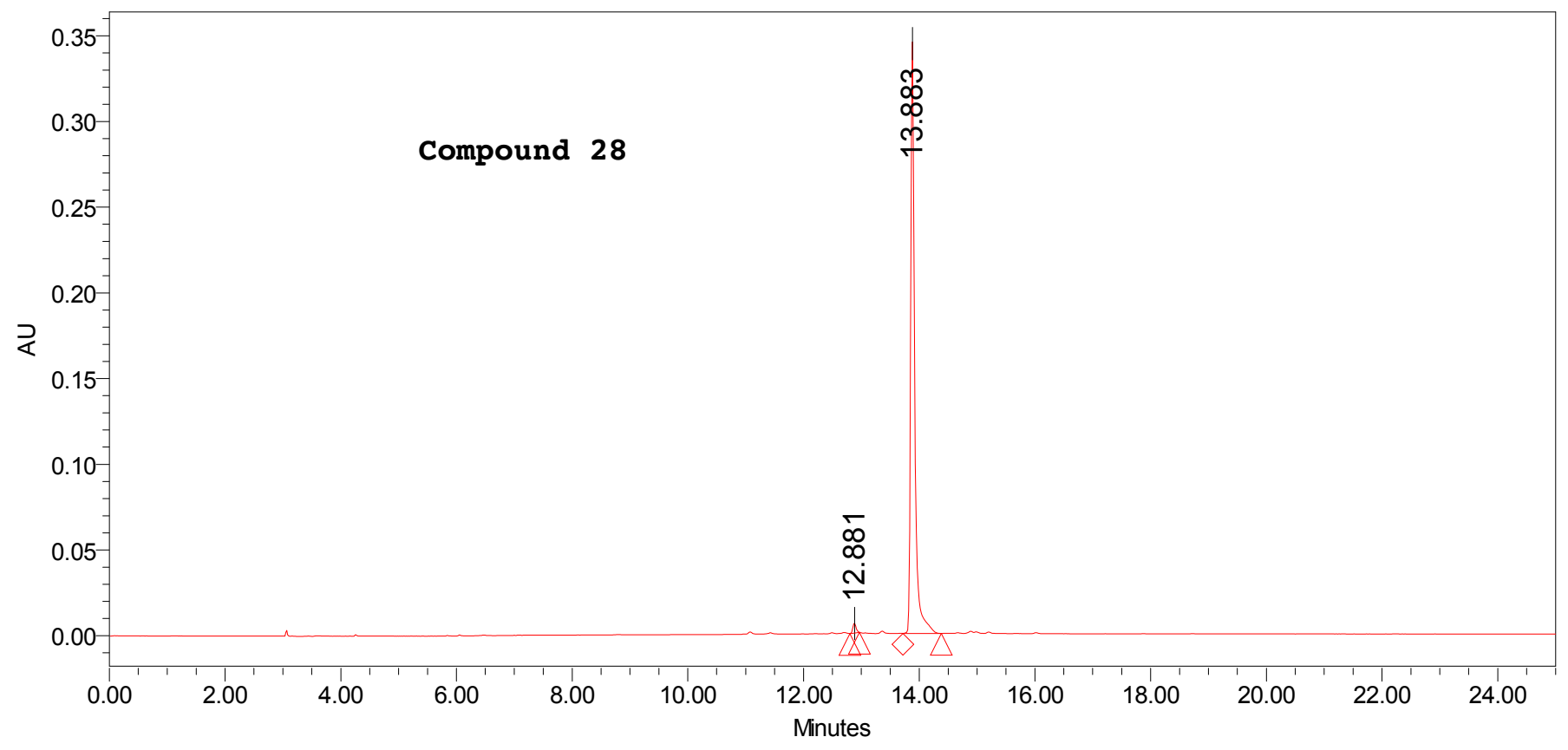

Peak Results
\begin{tabular}{|l|c|c|r|r|}
\hline & RT & Area & $\%$ Area & Height \\
\hline 1 & 12.881 & 22097 & 1.30 & 5511 \\
\hline 2 & 13.883 & 1680846 & 98.70 & 335519 \\
\hline
\end{tabular}

Reported by User: walkup Report Method: WalkUpReport Report Method ID 1018 Page: 1 of 1
Project Name: MedChemlMedChem2020-09

Date Printed:

$4 / 2 / 2021$ 


\begin{tabular}{|llll|}
\hline & \multicolumn{1}{c|}{ S A M P E } & I N F O R M A T I O N \\
\hline \hline Sample Name: & $14171-113$ & Acquired By: & walkup \\
Sample Type: & Unknown & Sample Set Name: & DZOXAgonist paper \\
Vial: & 106 & Acq. Method Set: & MethodSetAB5T95B15Min \\
Injection \#: & 1 & Processing Method: & 220W280W15MIN \\
Injection Volume: & 10.00 ul & Channel Name: & W2996 280.0nm-4.0 \\
Run Time: & 30.0 Minutes & Proc. Chnl. Descr.: & W2996 PDA 280.0 nm at 4.0 \\
& & & \\
Date Acquired: & $9 / 4 / 202011: 50: 09$ PM EDT & & \\
Date Processed: & $4 / 2 / 20214: 36: 56$ PM EST & & \\
\hline
\end{tabular}

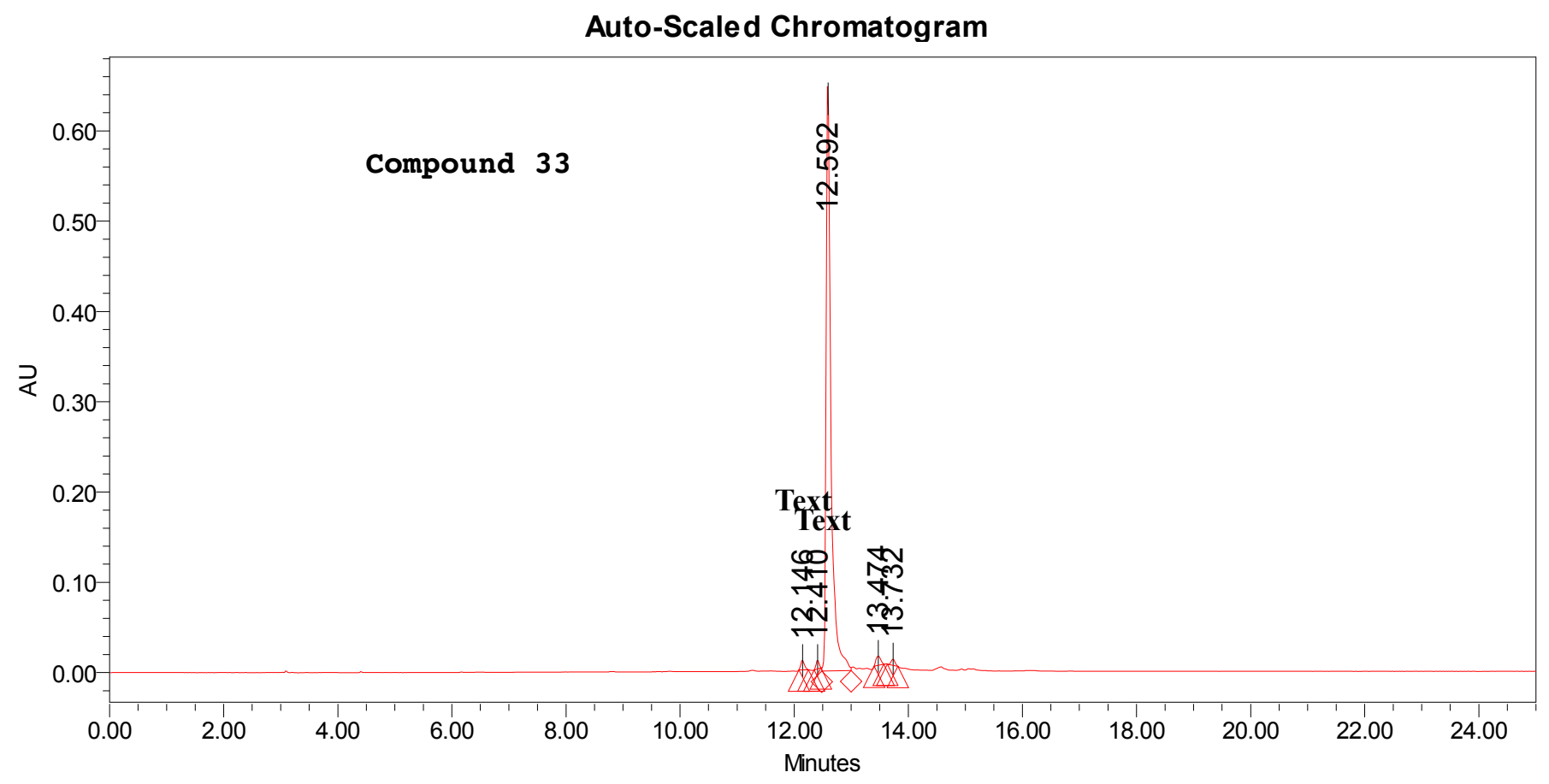

Peak Re sults
\begin{tabular}{|l|c|c|r|r|}
\hline & RT & Area & $\%$ Area & Height \\
\hline 1 & 12.146 & 47544 & 1.20 & 10379 \\
\hline 2 & 12.410 & 34343 & 0.87 & 9099 \\
\hline 3 & 12.592 & 3792449 & 95.63 & 646464 \\
\hline 4 & 13.474 & 58210 & 1.47 & 10043 \\
\hline 5 & 13.732 & 33009 & 0.83 & 6942 \\
\hline
\end{tabular}

Reported by User: walkup Report Method: WalkUpReport Report Method ID 1018 Page: 1 of 1
Project Name: MedChemlMedChem2020-09

Date Printed:

$4 / 2 / 2021$ 


\begin{tabular}{|llll|}
\hline & \multicolumn{1}{c|}{ S A M P L E } & I N F O R M A T I O N \\
\hline \hline Sample Name: & $14171-116$ & Acquired By: & walkup \\
Sample Type: & Unknown & Sample Set Name: & DZOXAgonist paper \\
Vial: & 109 & Acq. Method Set: & MethodSetAB5T95B15Min \\
Injection \#: & 1 & Processing Method: & 220W280W15MIN \\
Injection Volume: & 30.00 ul & Channel Name: & W2996 280.0nm-4.0 \\
Run Time: & 30.0 Minutes & Proc. Chnl. Descr.: & W2996 PDA 280.0 nm at 4.0 \\
& & & \\
Date Acquired: & $9 / 5 / 20201: 23: 09$ AM EDT & & \\
Date Processed: & $4 / 2 / 20214: 38: 39$ PM EST & & \\
\hline
\end{tabular}

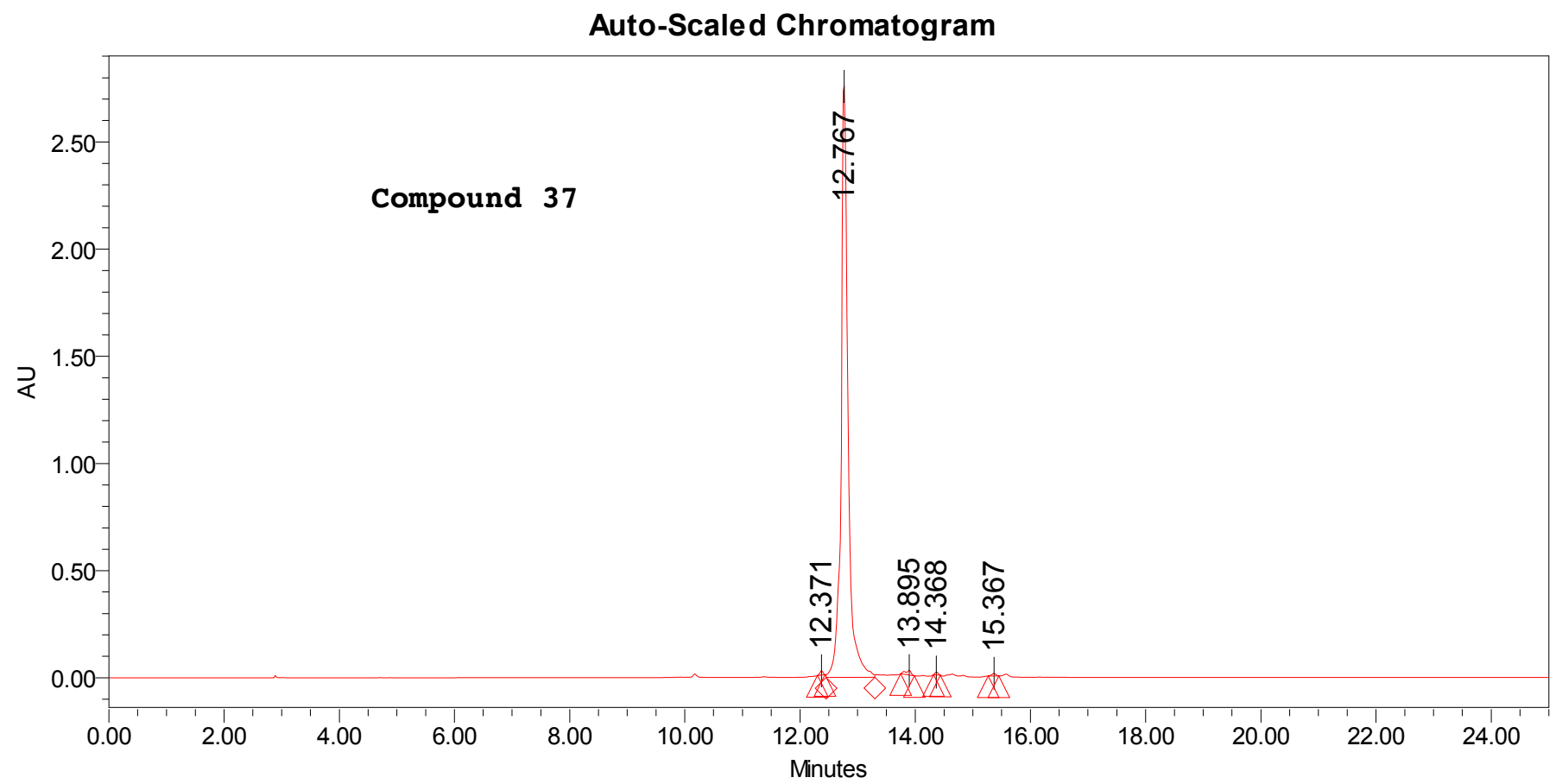

Peak Results
\begin{tabular}{|l|c|r|r|r|}
\hline & RT & \multicolumn{1}{|c}{ Area } & $\%$ Area & Height \\
\hline 1 & 12.371 & 75931 & 0.32 & 19529 \\
\hline 2 & 12.767 & 23094517 & 98.59 & 2789966 \\
\hline 3 & 13.895 & 145299 & 0.62 & 21135 \\
\hline 4 & 14.368 & 52928 & 0.23 & 13121 \\
\hline 5 & 15.367 & 56836 & 0.24 & 11613 \\
\hline
\end{tabular}

Reported by User: walkup Report Method: WalkUpReport Report Method ID 1018 Page: 1 of 1
Project Name: MedChemlMedChem2020-09

Date Printed:

$4 / 2 / 2021$ 
WalkUpReport

\begin{tabular}{|llll|}
\hline & \multicolumn{1}{c|}{ S A M P L E } & I N F O R M A T I O N \\
\hline \hline Sample Name: & $14171-143$ & Acquired By: & walkup \\
Sample Type: & Unknown & Sample Set Name: & DZOXAgonist paper \\
Vial: & 114 & Acq. Method Set: & MethodSetAB5T95B15Min \\
Injection \#: & 1 & Processing Method: & 220W280W15MIN \\
Injection Volume: & 30.00 ul & Channel Name: & W2996 280.0nm-4.0 \\
Run Time: & 30.0 Minutes & Proc. Chnl. Descr.: & W2996 PDA 280.0 nm at 4.0 \\
& & & \\
Date Acquired: & $9 / 5 / 2020$ 3:58:14 AM EDT & & \\
Date Processed: & $4 / 2 / 20214: 40: 01$ PM EST & & \\
\hline
\end{tabular}

\section{Auto-Scaled Chromatogram}

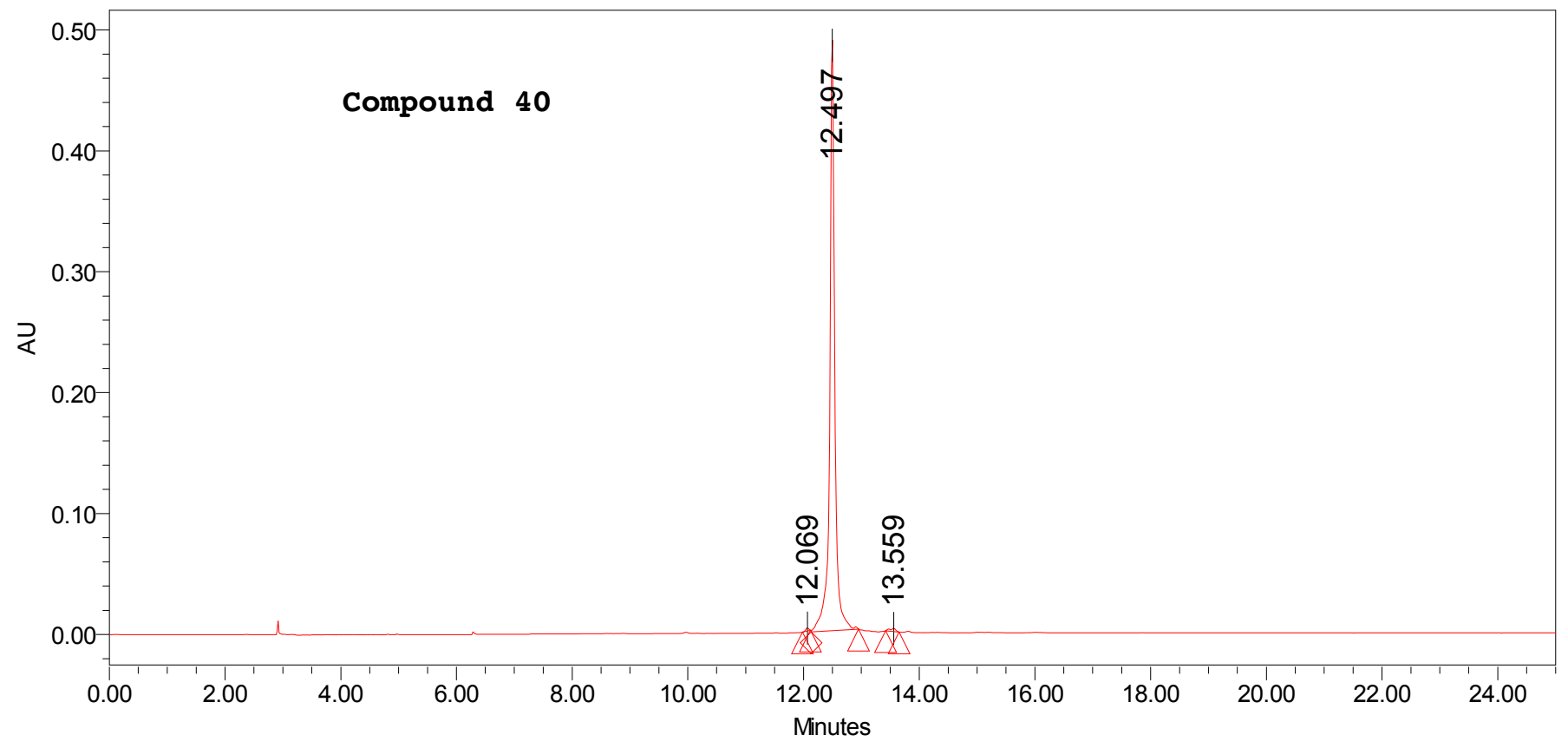

Peak Results

\begin{tabular}{|r|c|r|r|r|}
\hline & RT & Area & $\%$ Area & Height \\
\hline 1 & 12.069 & 9075 & 0.30 & 2385 \\
\hline 2 & 12.497 & 3007715 & 99.09 & 482507 \\
\hline 3 & 13.559 & 18681 & 0.62 & 2539 \\
\hline
\end{tabular}

Reported by User: walkup Report Method: WalkUpReport Report Method ID 1018 Page: 1 of 1
Project Name: MedChemlMedChem2020-09

Date Printed:

$4 / 2 / 2021$

S16 


\begin{tabular}{|llll|}
\hline & \multicolumn{1}{c|}{ S A M P L E } & I N F O R M A T I O N \\
\hline \hline Sample Name: & DZ14171-171 & Acquired By: & walkup \\
Sample Type: & Unknown & Sample Set Name: & 14171170195 \\
Vial: & 27 & Acq. Method Set: & MethodSetAB5T95B15Min \\
Injection \#: & 1 & Processing Method: & 220W280W15MIN \\
Injection Volume: & 10.00 ul & Channel Name: & W2996 280.0nm-4.0 \\
Run Time: & 30.0 Minutes & Proc. Chnl. Descr.: & W2996 PDA 280.0 nm at 4.0 \\
& & & \\
Date Acquired: & $8 / 31 / 20208: 13: 45$ PM EDT & & \\
Date Processed: & $4 / 2 / 20214: 46: 38$ PM EST & & \\
\hline
\end{tabular}

Auto-Scaled Chromatogram

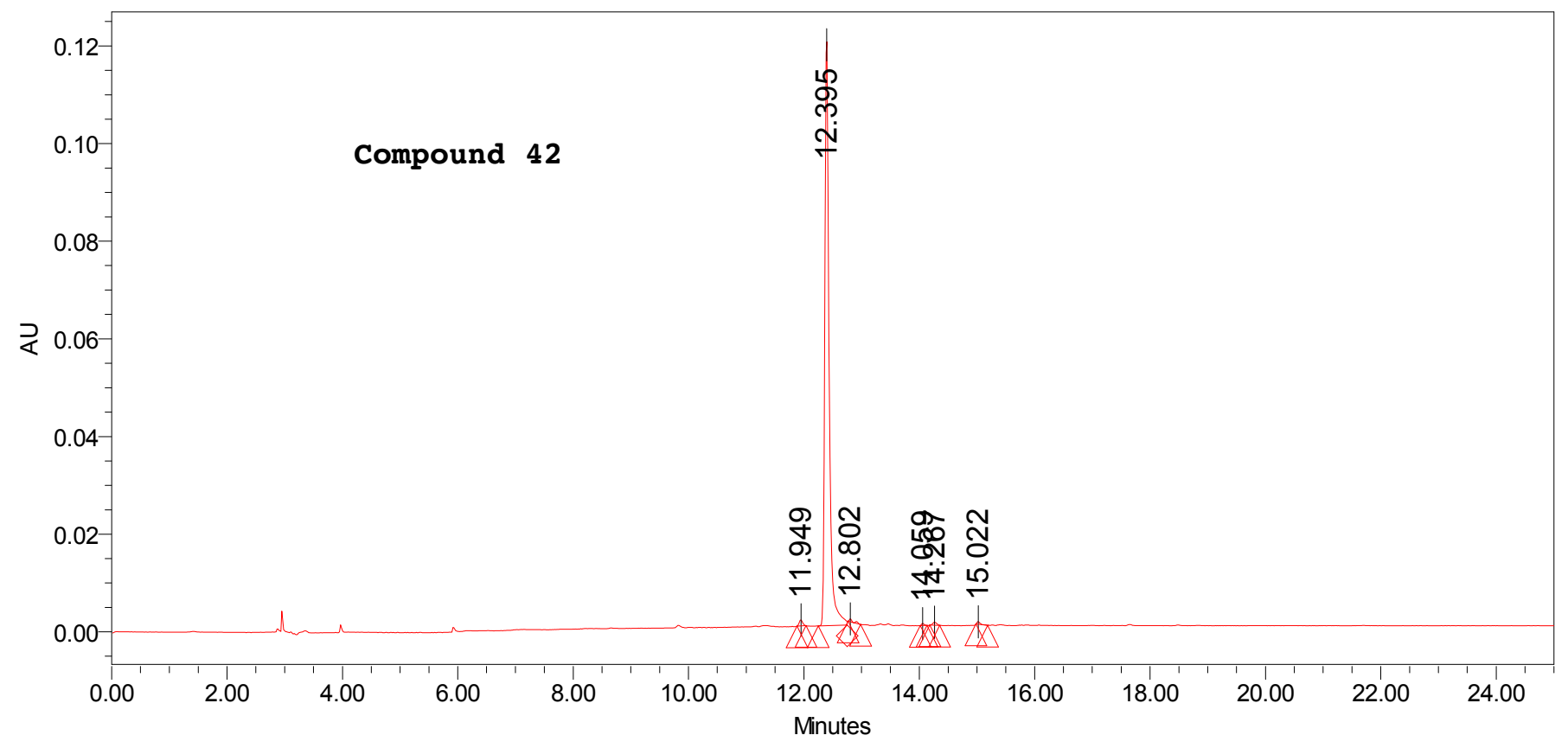

Peak Results

\begin{tabular}{|r|c|r|r|r|}
\hline & RT & Area & \% Area & Height \\
\hline 1 & 11.949 & 5247 & 0.82 & 1249 \\
\hline 2 & 12.395 & 625949 & 97.82 & 118736 \\
\hline 3 & 12.802 & 2897 & 0.45 & 561 \\
\hline 4 & 14.059 & 1235 & 0.19 & 356 \\
\hline 5 & 14.267 & 2749 & 0.43 & 596 \\
\hline 6 & 15.022 & 1850 & 0.29 & 500 \\
\hline
\end{tabular}




\begin{tabular}{|llll|}
\hline & \multicolumn{1}{c|}{ S A M P L E } & I N F O R M A T I O N \\
\hline \hline Sample Name: & DZ14171-195 & Acquired By: & walkup \\
Sample Type: & Unknown & Sample Set Name: & 14171170195 \\
Vial: & 28 & Acq. Method Set: & MethodSetAB5T95B15Min \\
Injection \#: & 1 & Processing Method: & 220W280W15MIN \\
Injection Volume: & 10.00 ul & Channel Name: & W2996 280.0nm-4.0 \\
Run Time: & 30.0 Minutes & Proc. Chnl. Descr.: & W2996 PDA 280.0 nm at 4.0 \\
& & & \\
Date Acquired: & $8 / 31 / 2020$ 8:44:46 PM EDT & & \\
Date Processed: & $4 / 2 / 20214: 49: 27$ PM EST & & \\
\hline
\end{tabular}

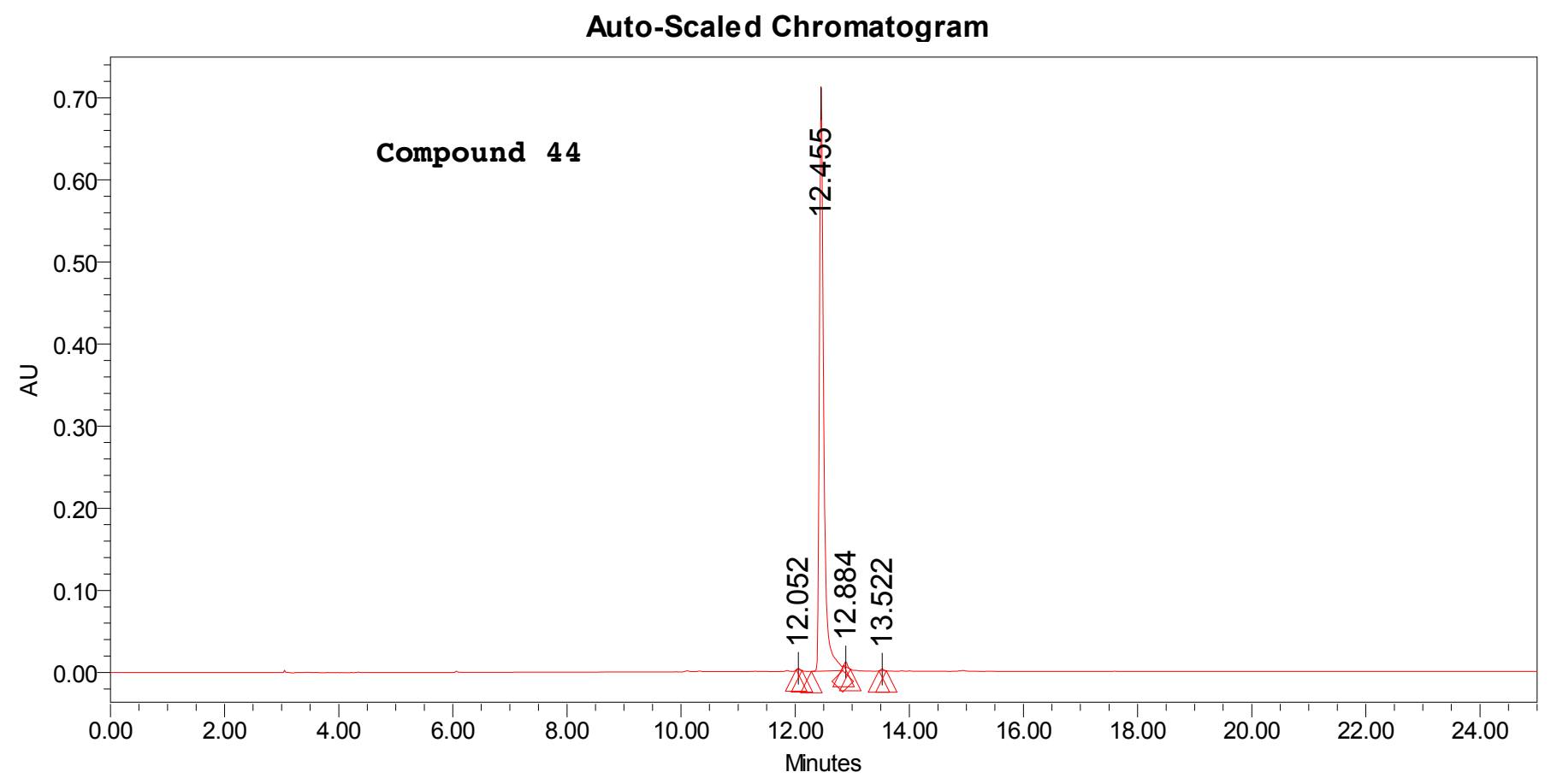

\begin{tabular}{|c|c|c|c|c|}
\hline \multicolumn{5}{|c|}{ Peak Results } \\
\hline & RT & Area & $\%$ Area & Height \\
\hline 1 & 12.052 & 7038 & 0.18 & 2102 \\
\hline 2 & 12.455 & 3786738 & 99.07 & 700017 \\
\hline 3 & 12.884 & 19634 & 0.51 & 5712 \\
\hline 4 & 13.522 & 8852 & 0.23 & 2185 \\
\hline
\end{tabular}

Reported by User: walkup Report Method: WalkUpReport Report Method ID 1018 Page: 1 of 1
Project Name: MedChemlMedChem2020-08

Date Printed:

$4 / 2 / 2021$ 


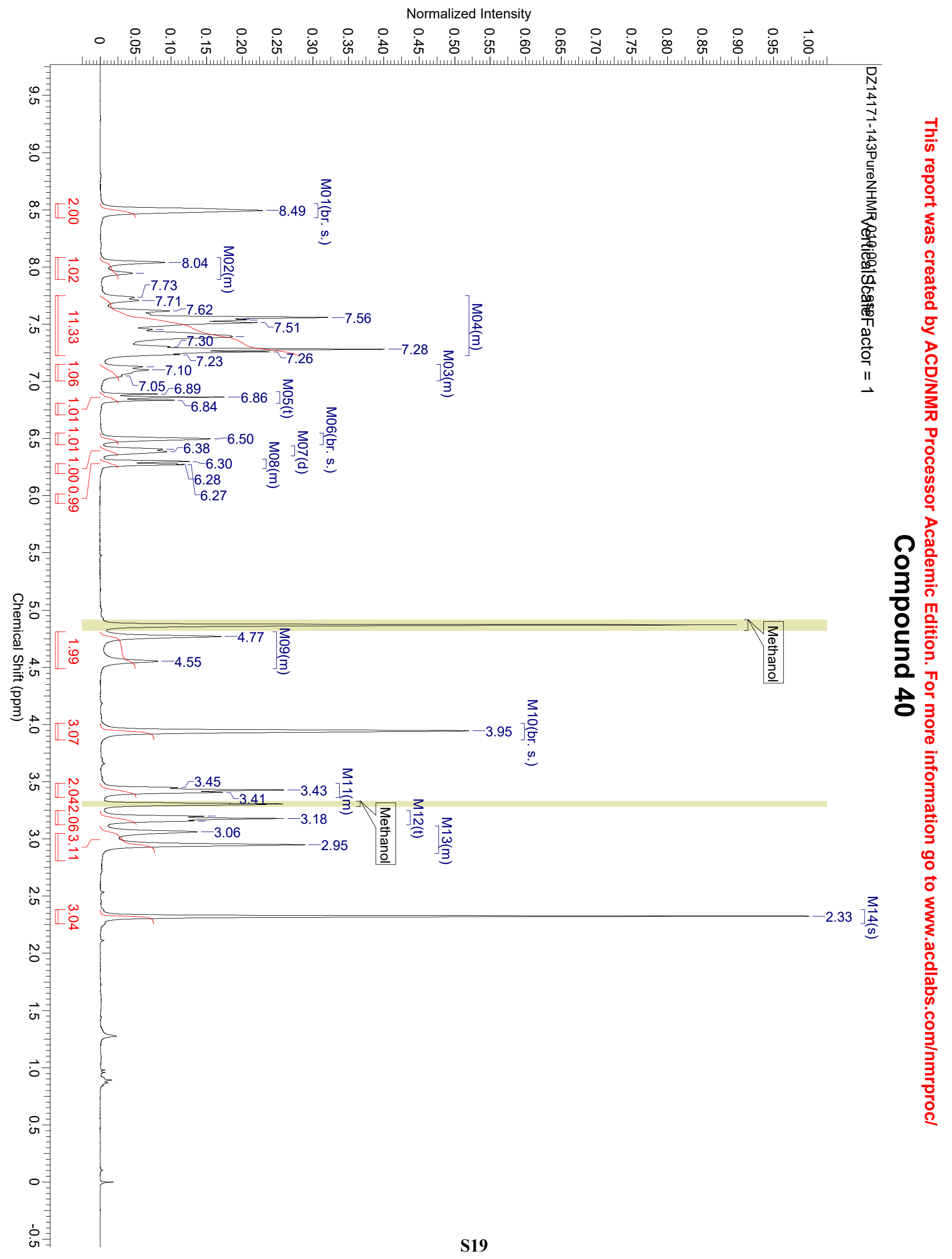




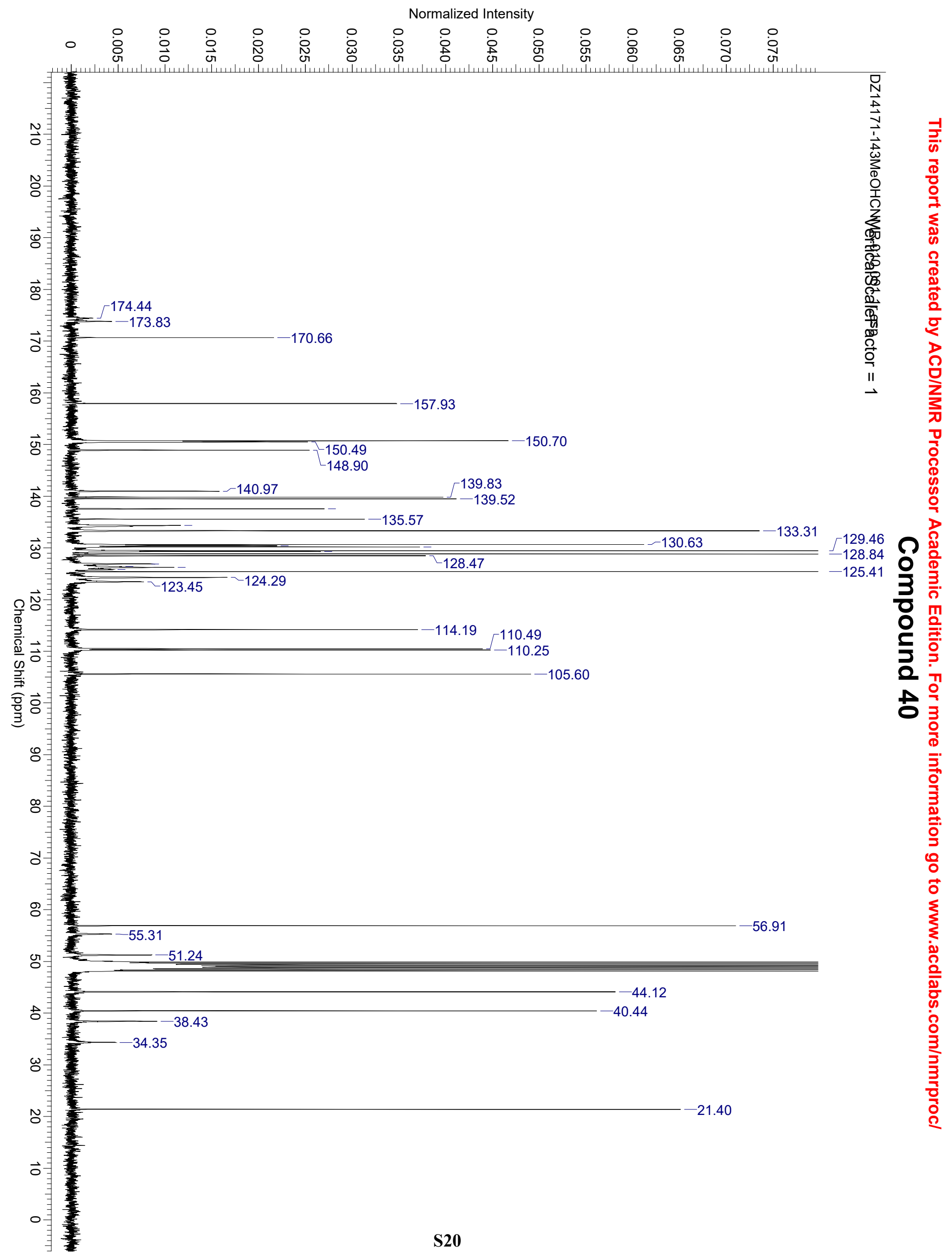

